Modeling, Identification and Control, Vol. 29, No. 3, 2008, pp. 103-115

\title{
Coordinator MPC for maximizing plant throughput *
}

\author{
Elvira Marie B. Aske ${ }^{2}$ Stig Strand ${ }^{2}$ Sigurd Skogestad ${ }^{1}$
}

\author{
${ }^{1}$ Department of Chemical Engineering, Norwegian University of Science and Technology, N-7491 Trondheim, \\ Norway.E-mail: Sigurd.Skogestad@chemeng.ntnu.no \\ ${ }^{2}$ StatoilHydro R\&DD, Process Control, N-7005 Trondheim, Norway. E-mail: \{elvira,stra\}@statoilhydro.com
}

\begin{abstract}
In many cases economic optimal operation is the same as maximum plant throughput, which is the same as maximum flow through the bottleneck(s). This insight may greatly simplify implementation. In this paper, we consider the case where the bottlenecks may move, with parallel flows that give rise to multiple bottlenecks and with crossover flows as extra degrees of freedom. With the assumption that the flow through the network is represented by a set of units with linear flow connections, the maximum throughput problem is then a linear programming (LP) problem. We propose to implement maximum throughput by using a coordinator model predictive controller (MPC). Use of MPC to solve the LP has the benefit of allowing for a coordinated dynamic implementation. The constraints for the coordinator MPC are the maximum flows through the individual units. These may change with time and a key idea is that they can be obtained with almost no extra effort using the models in the existing local MPCs. The coordinator MPC has been tested on a dynamic simulator for parts of the Kårst $\varnothing$ gas plant and performs well for the simulated challenges.
\end{abstract}

Keywords: bottleneck, maximize throughput, MPC

\section{Introduction}

Real-time optimization (RTO) offers a direct method of maximizing an economic objective function. Most RTO systems are based on detailed nonlinear steadystate models of the entire plant, combined with data reconciliation to update key parameters, such as feed compositions and efficiency factors in units, see for example Marlin and Hrymak (1997). Typically, the RTO application reoptimizes and updates on an hourly basis the set points for the lower-layer control system, which may consists of set points of local MPCs based on simple linear dynamic models. A steady-state RTO

\footnotetext{
${ }^{*}$ This article was originally published as: E.M.B. Aske, S. Strand and S. Skogestad, Coordinator MPC for maximizing plant throughput, Computers \& Chemical Engineering, 32, 195-204 (2008). Reprinted with permission from Elsevier.
}

is not sufficient if there are frequent changes in active constraints of large economic importance. For example, this could be the case if the throughput bottleneck in a plant moves frequently, which is the case for the application studied in this paper. At least in theory, it is then more suitable to use dynamic optimization with a nonlinear model, which may be realized using dynamic RTO (DRTO) or nonlinear MPC with an economic objective (Tosukhowong et al., 2004; Kadam et al., 2003; Strand, 1991). However, a centralized dynamic optimization of the entire plant is undesirable (Lu, 2003). An alternative is to use local unit-based MPCs, but the resulting steady-state target calculation may be far from optimal (Havlena and Lu, 2005). Coordination of multiple local MPCs have been studied by several authors. Cheng et al. (2004, 2006, 2007) have suggested to approach this "coordination" prob- 
lem by identifying appropriate interactions for linking constraints to find the steady-state targets for the local MPCs. Rawlings and Stewart (2007) discuss a cooperative distributed MPC framework, where the local MPC objective functions are modified to achieve systemwide control objectives. Ying and Joseph (1999) propose a two-stage MPC complement that track changes in the optimum caused by disturbances. The approach permits dynamic tracking of the optimum which is not achievable with a steady-state RTO used in conjunction with a single-stage MPC.

In this paper, we present a different and simpler solution that achieves economic optimal operation without any of these complexities. This solution applies to the common case where prices and market conditions are such that economic optimal operation of the plant is the same as maximizing plant throughput. The main objective is then to maximize the feed to the plant, subject to achieving feasible operation (satisfying operational constraints in all units). This insight may be used to implement optimal operation, without the need for dynamic optimization based on a detailed model of the entire plant.

From linear network theory, the max-flow min-cut theorem (Ford and Fulkerson, 1962) states that the maximum throughput in a linear network is limited by the "bottleneck(s)" of the network (Aske et al., 2007). In order to maximize the throughput, the flow at the bottlenecks should always be at their maximum. In particular, if the actual flow at the bottleneck is not at its maximum at any given time, then this gives a loss in production that can never be recovered (sometimes referred to as a "lost opportunity").

The throughput manipulators (TPMs) are the degrees of freedom available for implementing maximum throughput. They affect the flow through the entire plant (or at least in more than one unit), and therefore can not be used to control an individual unit or objective. Ideally, in terms of maximizing plant production and minimizing the back off, the TPM should be located at the bottleneck (Aske et al., 2007). However, the bottleneck may move depending on plant operating conditions (e.g. feed composition), and it is generally very difficult to change the TPM, once a decision on its location has been made. The reason is that the location of the TPM affects the degrees of freedom available for local control, and thus strongly affects the structure of the local control systems and in particular the structure of the inventory control system (Buckley, 1964; Price and Georgakis, 1993). The TPM will therefore generally be located away from the bottleneck, for example at the feed. For dynamic reasons it will then not be possible to achieve maximum flow through the bottleneck at all times, and a loss in production is in-

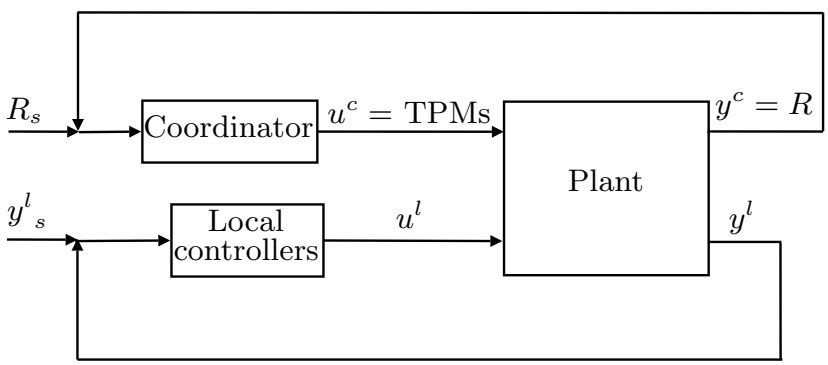

Figure 1: The coordinator uses the throughput manipulators $\left(u^{c}=\right.$ TPMs $)$ to control the remaining capacity $\left(y^{c}=R\right)$ in the units.

evitable.

The use of a coordinator controller that uses the throughput manipulators $\left(u^{c}=\mathrm{TPMs}\right)$ to control the remaining local capacity $\left(y^{c}=R=F_{\max }^{l}-F^{l}\right)$ in the units as illustrated in Figure 1. In the simplest case with a fixed bottleneck and feed rate as the TPM, the coordinator may be a single-loop PI-controller with the feed rate as the manipulated variable $\left(u^{c}\right)$ and the bottleneck flow as the controlled variable $\left(y^{c}\right)$, Skogestad (2004). However, more generally the coordinator must be a multivariable controller. Note from Figure 1 that the "coordinator" and the "local" controllers for the individual units are actually on the same level in the control hierarchy, like in decentralized control. Nevertheless, the term coordinator is used because the TPMs strongly affect all the units and because in general the coordinator controller must be designed based on a flow network model of the entire plant. An alternative to the decentralized structure is to combine all the local MPCs into a large combined MPC application that include the throughput manipulators as degrees of freedom.

Optimal operation corresponds to $R=0$ in the bottleneck, but if the maximum flow through the bottleneck is a hard constraint, then to avoid infeasibility $(R<0)$ dynamically, we need to "back off" from the optimal point

$$
\text { Back off }(b)=R_{s}=F_{\text {max }}^{l}-F_{s}^{l}
$$

More generally, the back off is the distance to the active constraint needed to avoid dynamic infeasibility in the presence of disturbances, model errors, delay and other sources for imperfect control (Narraway and Perkins, 1993; Govatsmark and Skogestad, 2005). The back off is a "safety factor" and should be obtained based on information about the disturbances and the expected control performance.

In this paper, we consider cases where the bottlenecks may move and with parallel trains that give rise to multiple bottlenecks and multiple throughput ma- 


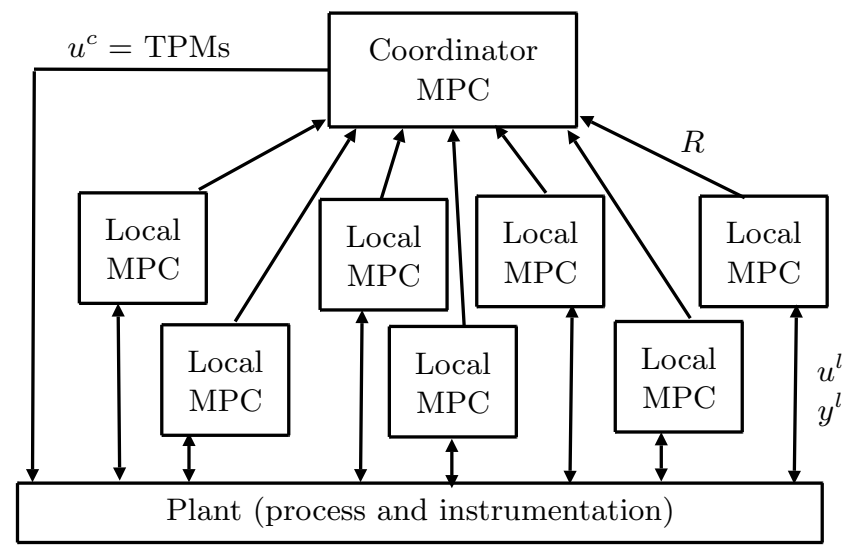

Figure 2: Proposed control structure where the coordinator MPC receives information from the local MPC about the remaining capacity $(R)$ in the units.

nipulators. This requires multivariable control and the proposed coordinator MPC both identifies the bottlenecks and implements the optimal policy. The constraints for the coordinator MPC are non-negative remaining capacities $(R \geq b \geq 0)$ in all units. The values of $R$ may change with time and a key idea is that they can be obtained with almost no extra effort using the existing local MPCs, as illustrated in Figure 2.

The paper is organized as follows. Economic optimal operation and the special case of maximum throughput is discussed in Section 2. Section 3 describes the coordinator MPC in addition to the capacity calculations in the local MPCs. Section 4 describes a dynamic simulation case study for a gas plant. A discussion follows in Section 5 before the paper is concluded in Section 6 .

\section{Maximum throughput as a special case of optimal operation}

Mathematically, the optimum is found by minimizing the cost $J$ (i.e., maximize the profit $(-J)$ ), subject to satisfying given specifications and model equations $(f=0)$ and operational constraints $(g \leq 0)$. At steadystate:

$$
\begin{array}{rl}
\min _{u} & J(x, u, d) \\
\text { s. t. } & f(x, u, d)=0 \\
g(x, u, d) \leq 0
\end{array}
$$

Here $u$ are the degrees of freedom (or manipulated variables, MVs), $d$ the disturbances and $x$ the (dependent) state variables. The degrees of freedom are split into those used for local control $\left(u^{l}\right)$ and the TPMs used for throughput coordinator $\left(u^{c}\right)$,

$$
u=\left[\begin{array}{l}
u^{l} \\
u^{c}
\end{array}\right]
$$

A typical profit function is

$$
(-J)=\sum_{j} p_{P_{j}} \cdot P_{j}-\sum_{i} p_{F_{i}} \cdot F_{i}-\sum_{k} p_{Q_{k}} \cdot Q_{k}
$$

where $P_{j}$ are the product flows, $F_{i}$ the feed flows, $Q_{k}$ the utility duties (heating, cooling, power), and $p$ denote the prices.

In many cases, and especially when the product prices are high, optimal operation of the plant (maximize $-J)$ is the same as maximizing throughput. To understand this, let $F$ denote the overall throughput in the plant, and assume that all feed flows are set in proportion to $F$,

$$
F_{i}=k_{F, i} F
$$

Then, under the assumption of constant efficiency in the units (independent of throughput) and assuming that all intensive (property) variables are constant, all extensive variables (flows and heat duties) in the plant will scale with the throughput $F$ e.g, Skogestad (1991). In particular, we have that

$$
P_{j}=k_{P, j} F ; Q_{k}=k_{Q, k} F
$$

where the gains $k_{P, j}$ and $k_{Q, k}$ and are constants. Note from (6) that the gains may be obtained from nominal (denoted 0) mass balance data:

$$
k_{P, j}=P_{j 0} / F_{0} ; k_{F, i}=F_{i 0} / F_{0} ; k_{Q, k}=Q_{k 0} / F_{0}
$$

Substituting (5) and (6) into (4) gives

$$
\begin{aligned}
& (-J)= \\
& \left(\sum_{j} p_{P_{j}} \cdot k_{P, j}-\sum_{i} p_{F_{i}} \cdot k_{F, i}-\sum_{k} p_{Q_{k}} \cdot k_{Q, k}\right) F \\
& =p F
\end{aligned}
$$

where $p$ is the operational profit per unit of feed $F$ processed. From the above derivation, $p$ is a constant for the case with constant efficiencies. We assume $p>0$ such that we have a meaningful case where the products are worth more than the feedstocks and utilities. Then, from (8) it is clear that maximizing the profit $(-J)$ is equivalent to maximizing the throughput $F$. However, $F$ cannot go to infinity, because the operational constraints $(g \leq 0)$ related to achieving feasible operation (indirectly) impose a maximum value for $F$.

In practice, the gains $k_{P, j}$ and $k_{Q, k}$ and are not constant, because the efficiency of the plant changes. Usually, operation becomes less efficient and $p$ decreases 
when $F$ increases. Nevertheless, as long as $p$ remains positive, $d(-J) / d F=p>0$ is nonzero, and we have a constrained optimum with respect to the throughput $F$. From (8) we see that $p$ will remain positive and optimal operation is the same as maximum throughput if the feed is available and product prices $p_{P, j}$ are sufficiently high compared to the prices of feeds and utilities.

\section{Coordinator MPC for maximizing throughput}

The overall feed rate (or more generally the throughput) affects all units in the plant. For this reason, the throughput is usually not used as a degree of freedom for control of any individual unit, but is instead left as an "unused" degree of freedom to be set at the plant-wide level. Most commonly, the throughput manipulators $\left(u^{c}\right)$ are set manually by the operator, but the objective here is to coordinate them to achieve economic optimal operation.

It is assumed that the local controllers (e.g. local MPCs) are implemented on the individual units. These adjust the local degrees of freedom $u^{l}$ such that the operation is feasible. However, local feasibility requires that the feed rate to the unit $F_{k}^{l}$ is below its maximum capacity, $F_{k, \max }^{l}$, and one of the tasks of the plant-wide coordinator is to make sure that this is satisfied. $F_{k, \text { max }}^{l}$ may change depending on disturbances (e.g. feed composition) and needs to be updated continuously. One method is to use the already existing models in the local MPCs, as discussed in Section 3.2.

\subsection{The coordinator MPC}

The steady-state optimization problem (2) can be simplified when the optimal solution corresponds to maximizing plant throughput. Consider the steady-state optimization problem

$$
\begin{aligned}
& \max _{u^{c}}(-J) \quad \text { s.t. } \\
& F^{l}=G u^{c} \\
& \quad R=F_{\text {max }}^{l}-F^{l} \geq b \geq 0 \\
& u_{\text {min }}^{c} \leq u^{c} \leq u_{\max }^{c}
\end{aligned}
$$

Here $F^{l}$ is a vector of local feeds to the units and $R$ is a vector of remaining capacities in the units. If the objective is to maximize throughput with a single feed, then $(-J)=F$. More generally, with different values of the feedstocks and products, the profit function in (4) is used. $G$ is a linear steady-state network model from the throughput manipulators $u^{c}$ (independent feed and crossover flows) to all the local flows $F^{l}$.
In order to achieve feasible flow through the network, it is necessary that $R \geq 0$ in all units. However, to guarantee dynamic feasibility, an additional back off from the capacity constraint may be required, which is represented by the vector $b$ in (11). The main difference from the original optimization problem (2) is that only $u^{c}$ (TPMs) are considered as degrees of freedom for the optimization in (9)-(12) and that the original constraints for the units $(f=0, g \leq 0)$ are replaced by a linear flow network and flow constraints $(R \geq b)$.

It is assumed that the local controllers generate close-to optimal values for the remaining degrees of freedom $u^{l}$, while satisfying the original equality ( $f=$ 0 ) and inequality constraints $(g \leq 0)$. This implies that no coordination of the local controllers is required, or more specifically that constant set points for the local controllers give close to optimal operation. In other words, it is assumed that we for the local units can identify "self-optimizing" controlled variables Skogestad (2000). If this is not possible then centralized optimization (RTO or maybe even DRTO) is required.

With the linear profit function $(-J)$ in $(4)$, the optimization problem in (9)-(12) is an LP problem. The optimal solution to an LP problem is always at constraints. This means that the number of active constraints in (11) and (12) must be equal to the number of throughput manipulators, $u^{c}$. Note that an active constraint in (11) corresponds to having $R_{k}=$ $F_{\text {max }, k}^{l}-F_{k}^{l}=b_{k}$, that is, unit $k$ is a bottleneck. This agrees with the max-flow min-cut theorem of linear network theory. However, to solve the LP problem, we will not make use of the max-flow min-cut theorem.

The steady-state optimization problem in (9)-(12) can be extended to the dynamic optimization problem:

$$
\begin{aligned}
\min _{u^{c}}\left(J-J_{s}\right)^{2} & +\Delta u^{c T} Q_{u} \Delta u^{c} \quad \text { s. t. } \\
F^{l} & =G_{d y n} u^{c} \\
R & =F_{\text {max }}^{l}-F^{l} \geq b \geq 0 \\
u_{\text {min }}^{c} & \leq u^{c} \leq u_{\max }^{c} \\
\Delta u_{\min }^{c} & \leq \Delta u^{c} \leq \Delta u_{\max }^{c}
\end{aligned}
$$

Maximum throughput under the presence of disturbances is dynamic in nature, and here, $G_{d y n}$ is a linear dynamic model from $u^{c}$ (manipulated variables, MVs) to the remaining capacity in each unit, $R_{k}$. Obtaining the dynamic models may be time consuming. However, it is possible to use simple mass balances to calculate the steady-state gains of $G_{d y n}$, see (7).

The dynamic cost function (13) includes penalty on the MV moves to ensure robustness and acceptable dynamic performance. The constraints are: back off on capacity to each unit (15), MV high and low limits (16) and MV rate of change limits (17). MV rate of change 
is mainly a safeguard for errors and is normally not used for tuning.

The term $\Delta u^{c T} Q_{u} \Delta u^{c}$ makes the objective function quadratic, whereas the objective function in the original problem (9) is linear. To obtain a quadratic objective function that fits directly into the MPC software used here, we have used a common trick of introducing a quadratic term $\left(J-J_{s}\right)^{2}$. The profit set point $J_{s}$ is high and unreachable with a lower priority than the capacity constraints. An alternative approach would be to include a linear term in $\mathrm{J}$ in (13).

Standard MPC implementations perform at each time step two calculations (Qin and Badgwell, 2003). First, the steady-state optimization problem with all the constraints is solved to obtain a feasible steadystate solution. Second, the dynamic problem is solved using the feasible targets obtained from the steadystate calculation. In our case, the steady-state part gives a feasible set point for the profit (or total flow) that replaces $J_{s}$ in the subsequent solution of the dynamic problem. The dynamic terms involving $\Delta u^{c}$ do not matter in the steady-state part, so the steady-state solution is identical to the LP problem in (9)-(12).

It is assumed that the local controllers (including local MPCs) are closed before obtaining the dynamic flow model $G_{d y n}$. To ensure stability, it is then advisable that the coordinator operates with a longer time horizon than the local MPCs.

\subsection{Capacity calculations using local MPCs}

An important parameter for the coordinator is the maximum flow for the individual (local) units, $F_{\text {max }}^{l}$. A key idea in the present work is to obtain updated values using on-line information (feedback) from the plant. Note that it is not critical that the estimate of the maximum capacity is correct, except when the unit is actually approaching its maximum capacity and the corresponding capacity constraint $R=F_{\max }^{l}-F^{l} \geq b$ becomes active. The use of on-line information from the actual plant will ensure that this is satisfied.

In simple cases, one may update the maximum capacity using the distance $(\Delta$ constraint $\geq 0)$ to a critical constraint in the unit,

$$
F_{\text {max }}^{l}=F^{l}+c \cdot \Delta \text { constraint }
$$

where $c$ is a constant and $F^{l}$ is the present flow through the unit. For example, for a distillation column $\Delta$ constraint $=\Delta p_{\max }-\Delta p$ could be difference between the pressure drop corresponding to flooding and the actual pressure drop.

In more complex cases, there may be more than one constraint that limits the operation of the unit and thus its maximum capacity. MPC is often implemented on the local units to improve dynamic performance and avoid complex logic. The maximum feed for each unit $k$ can then be easily estimated using the already existing models and constraints in the local MPC applications. The only exception may be that the model must be updated to include the feed to the unit, $F_{k}^{l}$, as an independent variable. The maximum feed to the unit $k$ is then obtained by solving the additional steady-state problem:

$$
F_{k, \max }^{l}=\max _{u_{k}^{l}, F_{k}^{l}} F_{k}^{l}
$$

subject to the linear model equations and constraints of the local MPC, which is a LP problem. Here $u_{k}^{l}$ is the vector of manipulated variables in the local MPC, and the optimization is subject to satisfying the linear constraints for the unit. To include past MV moves and disturbances, the end predictions of the variables should be used instead of the present values.

\section{Kårst $\varnothing$ gas processing case study}

The Kårst $\varnothing$ plant treats gas and condensate from central parts of the Norwegian continental shelf. The products are dry gas, which is exported through pipelines, and natural gas liquids (NGL) and condensate, which are exported by ships. The Kårstø plant plays a key role in the pipeline structure in the Norwegian Sea and therefore is maximum throughput usually the main objective. Also, from an isolated Kårstø point of view, the plant has relative low feed and energy costs and high product prices that favors high throughputs. There are no recycles in the plant. Usually, feed is available and can be manipulated within given limits.

The feed enters the plant from three different pipelines and the feed composition may change frequently in all three lines. Changes in feed compositions can move the main bottleneck from one unit to another and affect the plant throughput. The coordinator MPC approach has been tested with good results using the Kårstø Whole Plant simulator. This is a dynamic simulator built in the software D-SPICER).

\subsection{The case}

To demonstrate the applicability of the coordinator MPC, we use a detailed simulator model of parts of the Kårst $\varnothing$ plant. To avoid the need for large computer resources to run the process simulator, only parts of the whole plant are used in the case study, see Figure 3. The selected parts include two fractionation trains, T100 and T300. Both trains have a deethanizer, depropanizer, debutanizer and a butane splitter. In addition T300 has two stabilizers in parallel. There are six throughput manipulators $\left(u^{c}\right)$ as indicated by 


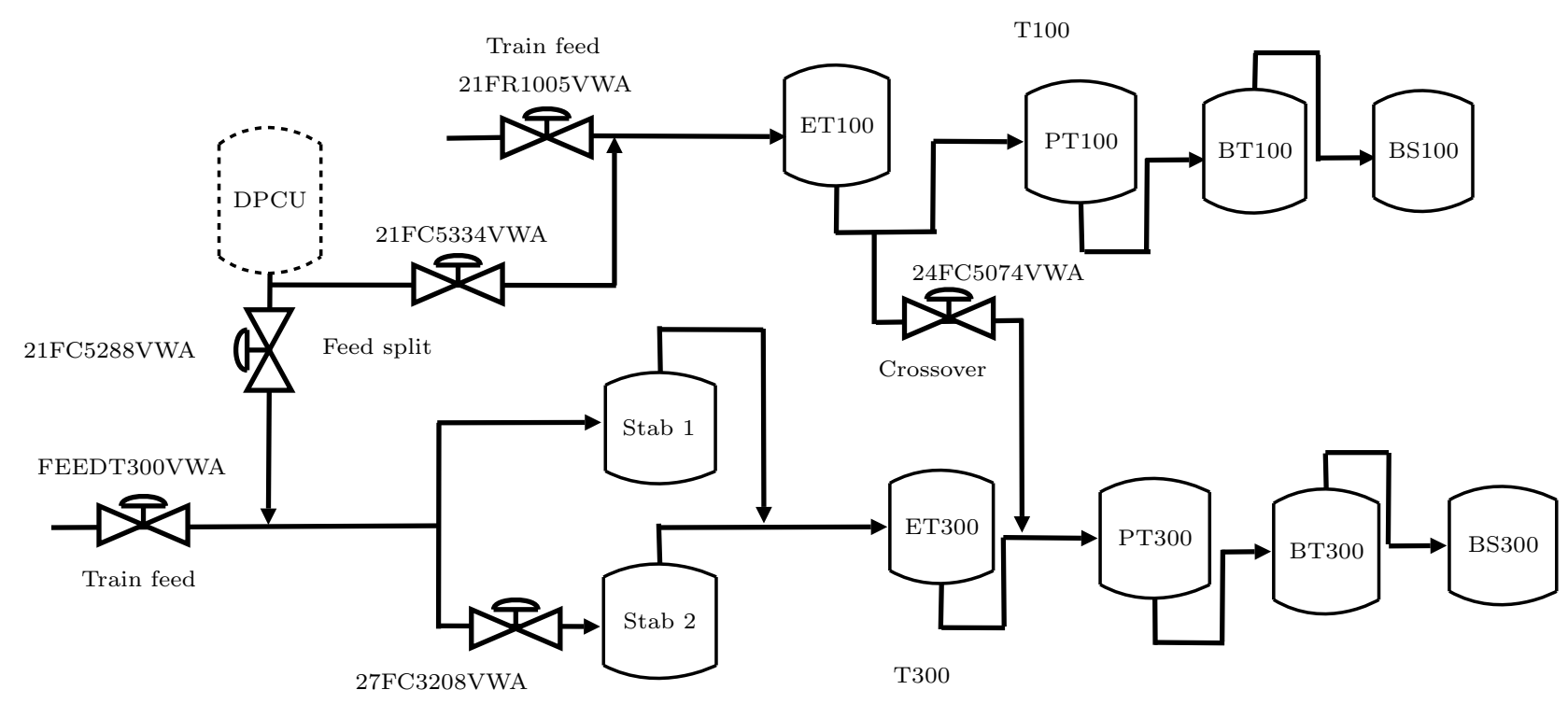

Figure 3: The simulated parts of the Kårstø plant

valves in Figure 3: two main train feeds, two liquid streams to the trains from the dew point control unit (DPCU), a crossover from train T100 to T300, and a flow split for the parallel stabilizers in train T300.

The local MPCs and the coordinator are implemented in Statoils SEPTIC ${ }^{1}$ MPC software (Strand and Sagli, 2003). Data exchange between the simulator and the MPC applications is done by the built-in D-SPICE® OPC server. The detailed dynamic simulator was used to obtain "experimental" step response models $\left(G_{d y n}\right)$ in the coordinator MPC. This approach has been found to work well in practice (Strand and Sagli, 2003).

\subsection{Implementation of the local MPCs}

The main control objective for each column is to control the quality in the top and bottom streams, by manipulating boil-up (V) and reflux flow (L). In addition the column must be kept under surveillance to avoid overloading, which is an important issue when maximizing throughput. Column differential pressure $(\Delta p)$ is used as an indicator of flooding (Kister, 1990). The remaining feed capacity for each column $\left(R_{k}\right)$ is calculated in the local MPC.

The LV-configuration with a temperature loop is used for regulatory control of the columns (Skogestad, 2007), and the local MPCs are configured as follows:

- CV (set point + constraint): Impurity of heavy key component

\footnotetext{
${ }^{1}$ Statoil Estimation and Prediction Tool for Identification and Control
}

- CV (set point + constraint): Impurity of light key component

- CV (constraint): Column differential pressure

- MV: Reflux flow rate set point

- MV: Tray temperature set point in lower section

- DV: Column feed flow

These MVs correspond to $u^{l}$ (local degrees of freedom), and CVs are the same as $y^{l}$. The feed rate is a disturbance variable (DV) for the local MPC, and is used as a degree of freedom when solving the extra LP problem to obtain the remaining capacity $(R)$ to be used by the coordinator. Some of the columns have additional limitations that are included as CVs in the local MPC. The product qualities are described as impurity of the key component and a logarithmic transformation is used to linearize over the operating region (Skogestad, 1997). The high limits on the product qualities are given by the maximum levels of impurity in the sales specifications and the differential pressure high limit is placed just below the flooding point.

The control specification priorities for solving the steady-state feasibility problem for the local MPC are as follows:

\section{High limit differential pressure}

2. Impurity limits

3. Impurity set points 
where 1 has the highest priority. The priority list is used in the steady state part in the MPC solver and leads to relaxation of the impurity set points (and in worst case limits) to avoid exceeding the differential pressure high limit (Strand and Sagli, 2003). By quality relaxation the column can handle the given feed rate without flooding the column. The low-priority quality set points are not used when solving the extra steadystate LP problem to obtain the remaining capacity $R$, because set point deviations are acceptable if the alternative is feed reduction. In the dynamic optimization part the constraints violations are handled by adding penalty terms to the objective function.

The local MPC applications are built with experimental step response models as described in Aske et al. (2005). The prediction horizon is 3 to 6 hours, which is significantly longer than the closed-loop response time. The sample time in the local MPC is set to 1 minute. From experience this is sufficiently fast for the distillation column applications and is the actual sample time used in the plant today.

\subsection{The design and implementation of the coordinator MPC}

The objective function for the coordinator is to maximize the total plant feed, $-J=F=\sum F_{i}$, which is the sum of the train feeds and the flows from the DPCU (FEEDT300VWA + 21FC5288VWA + 21FC5334VWA + 21FR1005VWA). The CVs and MVs for the coordinator MPC are:

- CV (high set point): Total feed flow $F$ to the plant (PLANT FEED).

- CVs (constraints): Remaining feed capacity $R_{k}$ in columns, 10 in total (R-ET100, R-PT100, RBT100, R-BS100, R-STAB1, R-STAB2, R-ET300, R-PT300, R-BT300, R-BS300)

- CV (constraint): T100 deethanizer sump level controller output (LC OUTLET)

- MV: Feed train 100 (21FR1005VWA)

- MV: Feed train 300 (FEEDT300VWA)

- MV: Feed from DPCU to train 100 (21FC5334VWA)

- MV: Feed from DPCU to train 300 (21FC5288VWA)

- MV: Crossover flow from T100 to T300 (24FC5074VWA)

- MV: Stabilizers feed split (27FC3208VWA)
These MVs correspond to $u^{c}$ (coordinator degrees of freedom). The deethanizer sump level controller output CV (gives the feed to PT100) is used to avoid emptying or overfilling up the sump level in ET100 when manipulating the crossover. The total plant feed has a high unreachable set point with low priority. The remaining feed capacity low limits, and high and low limits of the level controller output have high priority.

Note that each train has two feeds; one train feed and one from the dew point control unit (DPCU). The two feeds have different compositions, and this makes it possible for the coordinator to adjust the feed composition, and thus adjust the load to specific units. The two stabilizers are identical in the simulator, so the stabilizer split (27FC3208VWA) will ensure equal load to the stabilizers. The coordinator uses experimental step response models, obtained in the same way as for the local MPCs. The models were obtained at $80-95 \%$ of the maximum throughput, which is typical for the current plant operation. The coordinator execution rate is slower than in the local MPCs to ensure robustness and is here chosen to be 3 minutes. The prediction horizon is set to 20 hours.

The coordinator attempts to maximize the total feed rate while satisfying the capacity constraints for the units. Since the capacity constraints are "hard", it is necessary to introduce at steady-state a back off $b$ to ensure $R \geq 0$ also dynamically. Tuning of the coordinator MPC is a trade-off between robustness and MV (feed) variation on the one side and keeping the flows through the bottlenecks close their maximum on the other side. The required back off $b$ needs to be obtained after observing over some time the performance of coordinator MPC. In the case study, the value of $b$ is about $1-2 \%$ of the feed to the unit.

\subsection{Results from the simulator case study}

The coordinator MPC performance is illustrated with three different cases:

1. Take the plant from unconstrained operation (with given feed rate) to maximum throughput (at $t=0$ $\min )$

2. Change in feed composition (at $t=360 \mathrm{~min}$ )

3. Change in a CV limit in a local MPC (at $t=600$ $\min$ )

All three cases are common events at the Kårstø plant. Feed composition changes are the most frequent ones. The coordinator should also be able to handle operator changes in the local MPCs as illustrated by changing a local CV limit. 
The most important CVs in the coordinator MPC are displayed in Figure 4 and the corresponding coordinator MVs are shown in Figure 5. CVs far from their constraints are omitted. The vertical lines in the Figures indicate the time where disturbances are introduced (Cases 2 and 3). The back off from the capacity constraints is indicated by dashed horizontal lines in Figure 4. Figure 6 shows the response of a local MPC application (BS100).

\subsubsection{Case 1: Take the plant to maximum throughput}

Initially, the plant is not operating at maximum throughput, and Figure 5 shows that all four feed rates are ramped up over the first hour. The crossover (24FC5074VWA in Figure 5) is reduced to unload train 300 where BS300 is close to its capacity limit even initially (the plant is not steady state at $t=0$ min). From Figure 4, ET100 and the T300 stabilizers (Stab1 and Stab2) impose a bottleneck upstream of the crossover, whereas BS300 is a bottleneck downstream the crossover, at least for some period. The remaining capacity in BS300 violates its lower limit of $b=1.6 \mathrm{t} / \mathrm{h}$, and is actually just below zero for some time. Hence the back off $b$ is not sufficiently large to keep the remaining capacity just above zero in this case. From Figure 6, we see that the local MPC application for BS100 relaxes the quality set points because the column reaches the differential pressure high limit.

\subsubsection{Case 2: Change in feed composition}

A feed composition step change is introduced to the train 100 feed (sum of 21FR1005VWA and 21FC5335VWA). The composition change is given in Table 1 and occurs at time $t=360$ minutes, at the first vertical line in Figures 4, 5 and 6. The reduction in ethane content leads to an increase in the remaining feed capacity in ET100, which is a bottleneck at that time, and the coordinator can increase the train feed. However, the increase in iso-butane content reduces the remaining feed capacity in the further downstream butane splitter (BS100), which becomes a new bottleneck. The coordinator increases the crossover to make use of some remaining capacity in train 300 .

\subsubsection{Case 3: Change in a CV limit in a local MPC}

The bottom quality high limit in BS100 is reduced at a time where BS100 is already operating at its capacity limit, as can be seen at $t=600$ minutes in Figure 6 . This leads to a reduction in the remaining feed capacity in BS100 of about $2 \mathrm{t} / \mathrm{h}$. The coordinator MPC responds by increasing the crossover flow from T100
Table 1: The feed composition change in the T100 feed at $t=360$ minutes

\begin{tabular}{ccc}
\hline Component & Nominal $[\mathrm{mol} \%]$ & Points change [\%] \\
\hline Ethane & 37.3 & -1.1 \\
Propane & 35.4 & 0.71 \\
Iso-butane & 5.64 & 5.6 \\
N-butane & 11.3 & -0.34 \\
Iso-pentane & 1.79 & 0.09 \\
N-pentane & 1.79 & 0.10 \\
\hline
\end{tabular}

to T300 in addition to T100 feed reduction. The two butane splitters (BS100 and BS300) are now the bottlenecks together with the stabilizers. As expected, the overall effect of the stricter quality limit is reduction in the total plant feed. The reduction takes a long time, however, because the bottleneck in the butane splitters is quite far from the plant feeds.

\section{Discussion}

The main assumption behind the proposed coordinator MPC (see (13)-(17)), is that optimal operation corresponds to maximum throughput. This will always be the case if the flow network $\left(G_{d y n}\right)$ is linear because we then have a LP problem. However, as discussed in Section 2, even a nonlinear network will have maximum throughput as the optimal solution provided the product prices are sufficiently high. Thus, the use of a linear flow network model $\left(G_{d y n}\right)$ in the coordinator MPC is not a critical assumption. The coordinator identifies the maximum throughput solution based on feedback about the remaining capacity in the individual units, and the main assumption for the network model is that the gains (from feed rates to remaining capacities) have the right sign. Nevertheless, a good network model, both static and dynamic, is desired because it improves the dynamic performance of the coordinator MPC.

In this application, the remaining capacity is obtained for individual units. However, in some cases, for example, reactor-recycle systems, it may be better to consider system bottlenecks caused by the combination of several units (Aske et al., 2007).

By using a decoupled strategy based on the remaining feed capacity in each unit, the coordinator MPC exploits the already existing models in the local MPCs. This leads to a much smaller modelling effort compared to alternative approaches, like RTO based on a detailed nonlinear model of the entire plant. The computation time in the coordinator MPC is small, and facilitates fast corrections of disturbances, model errors and transient dynamics. The coordinator MPC 

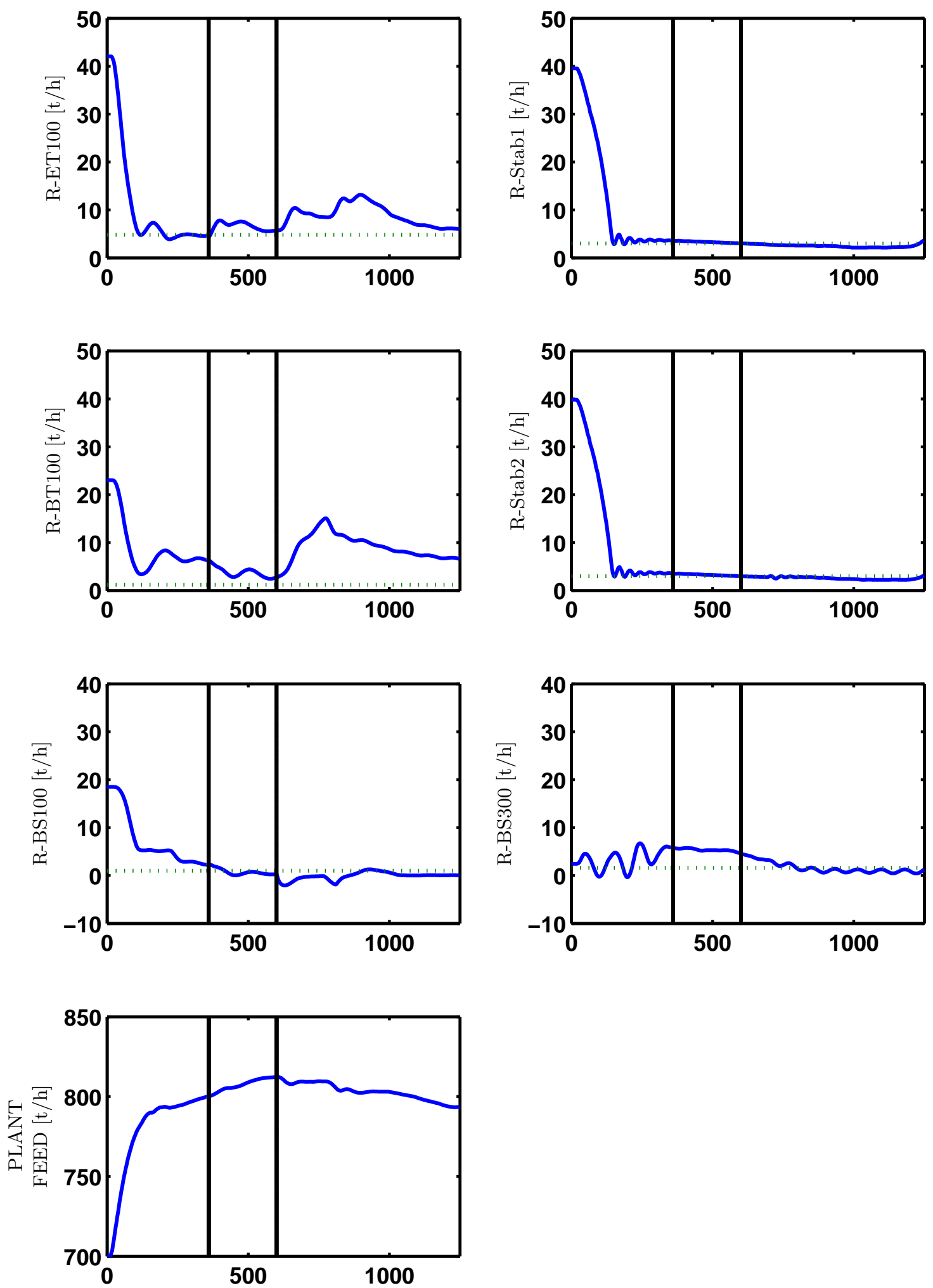

minutes

Figure 4: The most important CVs in the coordinator MPC (solid) with CV limits (dotted) 
T100

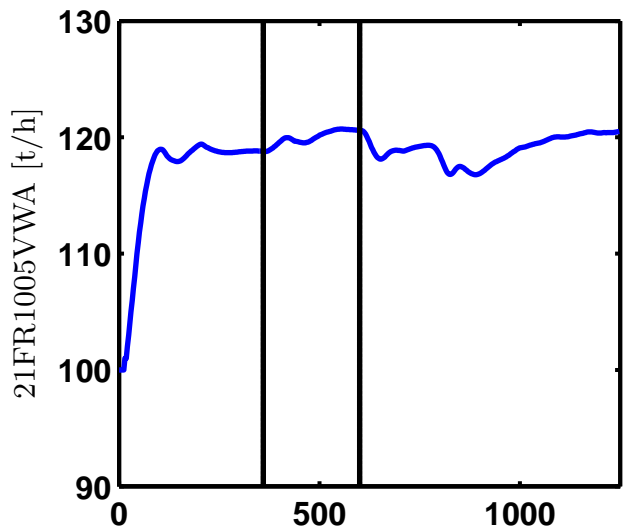

DPCU-T100
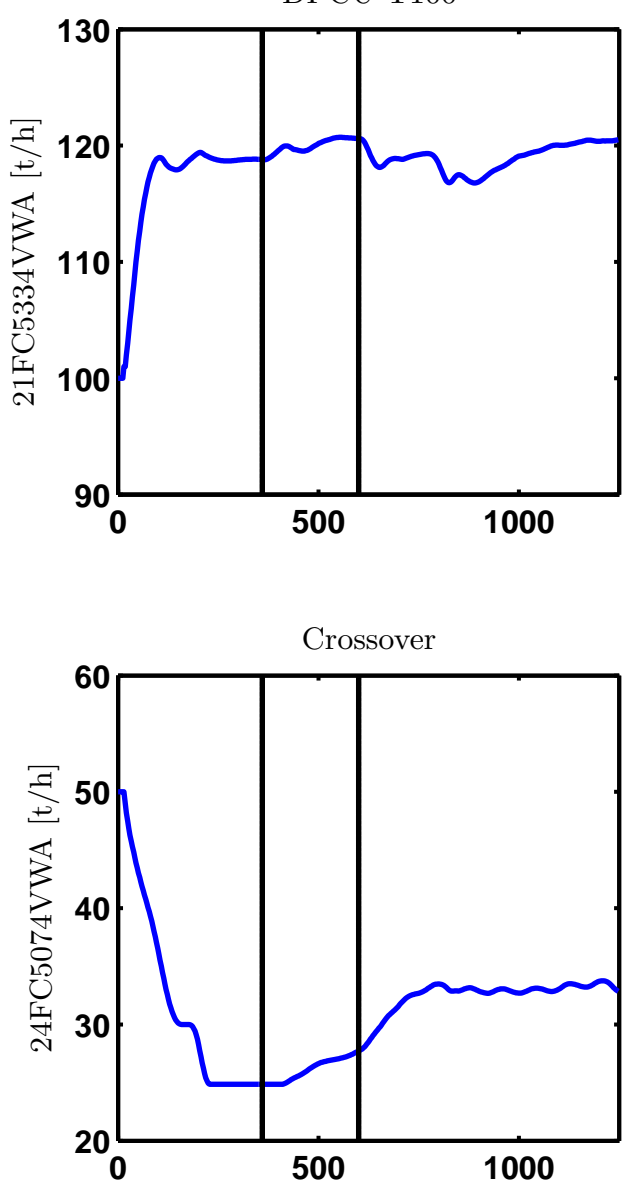

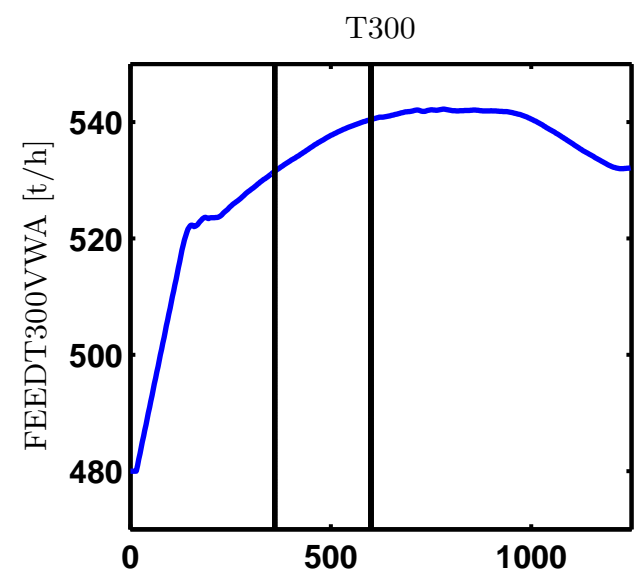

DPCU-T300

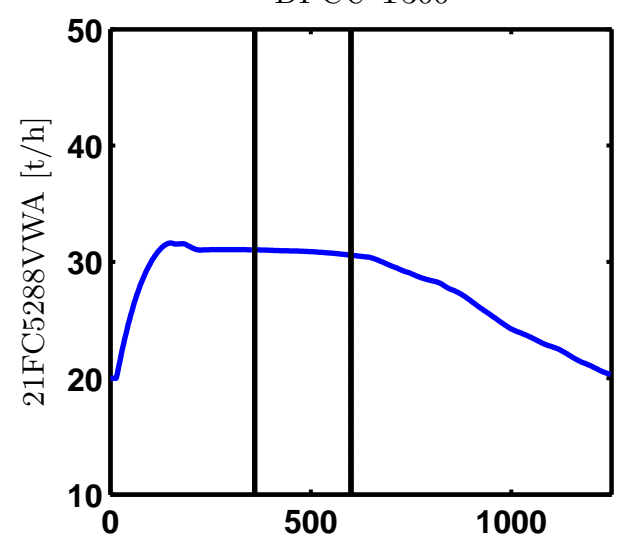

minutes

Figure 5: MVs in the coordinator MPC. Vertical lines indicate new case. 

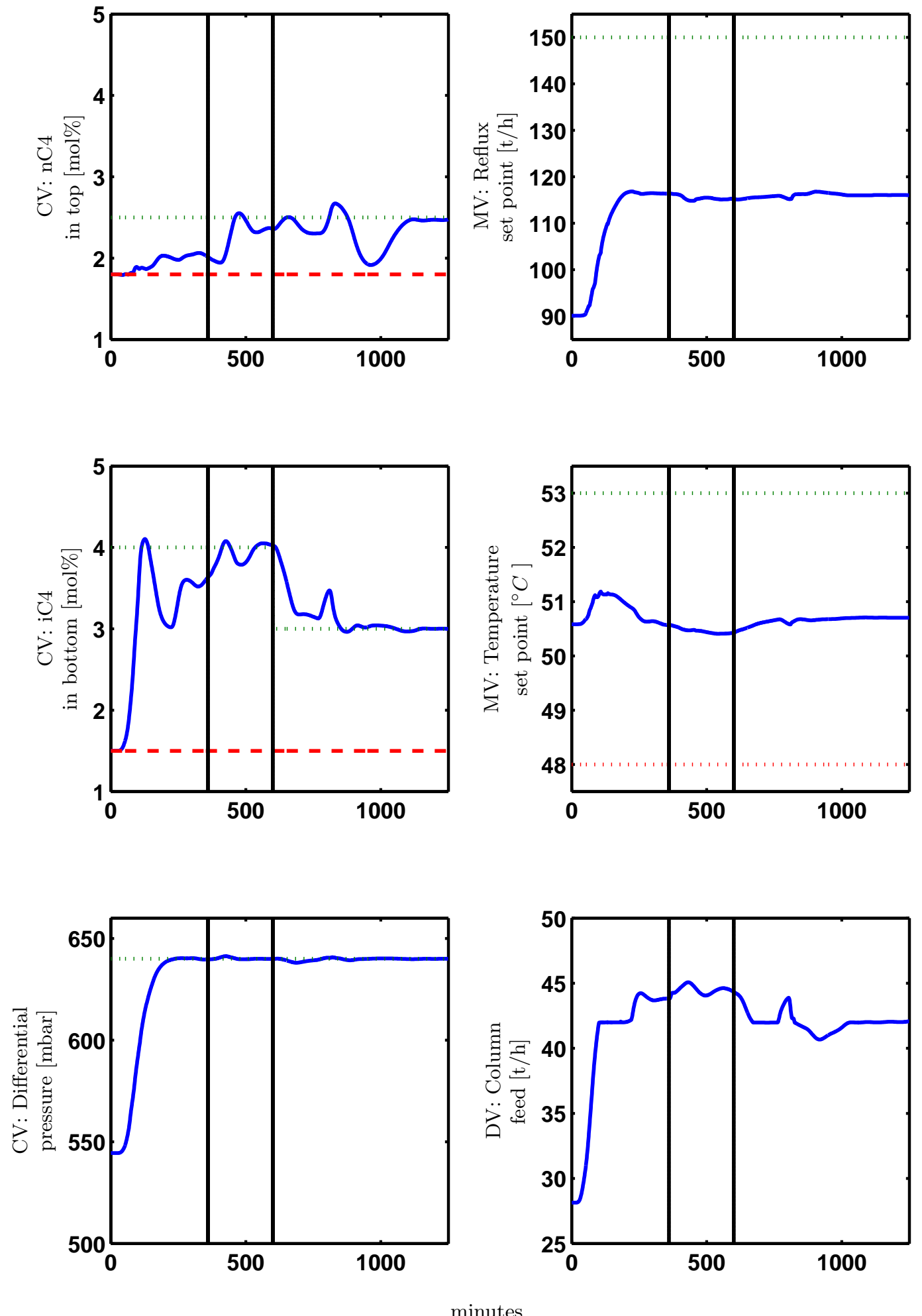

Figure 6: CVs, MVs and DV in BS100 MPC. Horizontal lines are set points (dashed) and limits (dotted). 
effectively solves the DRTO problem with acceptable accuracy and execution frequency.

An alternative coordinator MPC strategy would be to combine all the local MPCs into one large combined MPC application including the throughput manipulators. However, for a complete plant the application will be over-complex leading to challenging modelling and maintenance. The improvement by using a combined approach compared to our simple coordinator MPC is expected to be minor since the set points to the MPC are not coordinated. Set point coordination would require a nonlinear model for the entire plant, for example, RTO.

A back off from the maximum throughput in the units is necessary due to unmeasured disturbances and long process response times. The back off should be selected according to the control performance and acceptable constraint violations. In general, the back off can be reduced by improving the dynamic network model and including more plant information to allow for feed-forward control. For example, feed composition changes could be included in the coordinator MPC to improve performance. Due to the lack of fast and explicit feed composition measurements in the plant, feed composition changes are treated as unmeasured disturbances in the simulations in the current concept. However, the concept can be extended by using intermediate flow measurements as indicator for feed composition changes. Therefore, the use of alternative model structures that will simplify and propagate model corrections from intermediate flow measurements should be evaluated.

The most effective way of reducing the back off is to introduce throughput manipulators that are located closer to the bottlenecks. This reduces the dynamic response time and gives tighter control of the flow through the bottleneck. In the case study, the crossover flow introduces a throughput manipulator in the middle of the plant, which improves the throughput control of the units downstream the crossover. It is also possible to include additional dynamic throughput manipulators that make use of the dynamic buffer capacity in the various units and intermediate tanks in the network.

The coordinator requires that the local MPC are well tuned and work well. If the local MPC is not well tuned, a larger back off is needed to avoid constraint violation in the coordinator MPC. In the case study, the BS300 MPC should be retuned to give less oscillations at high throughputs.

The term "coordinator" is used by some authors (Venkat, Rawlings, and Wright and Cheng, Forbes, and Yip) to describe coordination of multiple MPCs where the coordinator is at the level above and generates set points to the local MPCs. In this work the term "coordinator" is used in the meaning of coordinating the flow through the plant, and the coordinator at the same level in the control hierarchy as the local MPCs (see Figure 1). However, the tuning is assumed to be done sequentially, with the local MPCs being closed before obtaining the flow network model and tuning the coordinator MPC.

\section{Conclusion}

In many cases, optimal operation is the same as maximum throughput. In terms of realizing maximum throughput there are two issues, first identifying bottleneck(s) and second, implementing maximum flow at the bottleneck(s). The first issue is solved by using the models and constraints from the local unit MPC applications to obtain an estimate of the remaining feed capacity of each unit. The second issue is solved using a standard MPC framework with a simple linear flow network model. The overall solution is a coordinator MPC that manipulates on plant feeds and crossovers to maximize throughput. The coordinator MPC has been tested on a dynamic simulator for parts of the Kårst $\varnothing$ gas plant, and it performs well for the simulated challenges.

\section{References}

Aske, E., Skogestad, S., and Strand, S. Throughput maximization by improved bottleneck control. In 8th International Symposium on Dynamics and Control of Process Systems (DYCOPS), volume 1. Cancun, Mexico, 2007 pages 63-68.

Aske, E., Strand, S., and Skogestad, S. Implementation of MPC on a deethanizer at Kårstø gas plant. In 16th IFAC World Congress, paper WeM06-TO/2. Prague, Czech Republic, 2005 pages CD-rom published by International Federation of Automatic Control.

Buckley, P. S. Techniques of Process Control. John Wiley \& Sons, Inc., NY, USA, 1964.

Cheng, R., Forbes, J., and Yip, W. Dantzig-Wolfe decomposition and large-scale constrained MPC problems. In International Symposium on Dynamics and Control of Process Systems (DYCOPS). Boston, USA, 2004 pages paper 117, in CD rom.

Cheng, R., Forbes, J., and Yip, W. Coordinated decentralized MPC for plant-wide control of a pulp mill benchmark problem. In International Symposium on Advanced Control of Chemical Processes 
(ADCHEM), volume 2. Gramado, Brazil, 2006 pages 971-976.

Cheng, R., Forbes, J., and Yip, W. Price-driven coordination method for solving plant-wide MPC problems. J. Proc. Control, 2007. 17:429-438.

Ford, L. and Fulkerson, D. Flows in Networks. Princeton University Press, 1962.

Govatsmark, M. and Skogestad, S. Selection of controlled variables and robust setpoints. Ind. Eng. Chem. Res., 2005. 44:2207-2217.

Havlena, V. and $\mathrm{Lu}$, J. A distributed automation framework for plant-wide control, optimisation, scheduling and planning. In P. Horacek, M. Simandl, and P. Zitek, editors, 16th Triennial World Congress of the International Federation of Automatic Control. Prague, Czech Republic, 2005 pages 80-94.

Kadam, J., Marquardt, W., Schlegel, M., Backx, T., Bosgra, O., Brouwer, P.-J., Dünnebier, G., van Hessem, D., Tiagounov, A., and de Wolf, S. Towards integrated dynamic real-time optimization and control of industrial processes. In Proceedings Foundations of Computer-Aided Process Operations (FOCAPO2003). Coral Springs, Florida, 2003 pages 593-596.

Kister, H. Z. Distillation Operation. McGraw Hill, NY, USA, 1990.

Lu, J. Challenging control problems and emerging technologies in enterprise optimization. Control Engineering Practice, 2003. 11:847-858.

Marlin, T. E. and Hrymak, A. N. Real-time operations optimization of continuous processes. In J. Kantor, C. Garcia, and B. Carnahan, editors, Fifth International Conference on Chemical Process Control (CPC-5). Lake Tahoe, Nevada, 1997 pages 156-164.

Narraway, L. and Perkins, J. Selection of process control structure based on linear dynamic economics. Ind. Eng. Chem. Res., 1993. 32(11):2681-2692.

Price, R. M. and Georgakis, C. Plantwide regulatory control design procedure using a tiered framework. Ind. Eng. Chem. Res., 1993. 32:2693-2705.

Qin, S. and Badgwell, T. A survey of industrial model predictive control technology. Control Engineering Practice, 2003. 11:733-764.

Rawlings, J. and Stewart, B. Coordinating multiple optimization-based controllers: New opportunities and challenges. In 8th International Symposium on Dynamics and Control of Process Systems (DY$C O P S)$, volume 1. Cancun, Mexico, 2007 pages 1928.

Skogestad, S. Consistency of steady-state models using insights about extensive variables. Ind. Eng. Chem. Res., 1991. 30:654-661.

Skogestad, S. Dynamics and control of distillation columns - A tutorial introduction. Trans. IChemE, 1997. 75(Part A):539-562.

Skogestad, S. Self-optimizing control: the missing link between steady-state optimization and control. Comput. Chem. Eng., 2000. 24:569-575.

Skogestad, S. Control structure design for complete chemical plants. Comput. Chem. Eng., 2004. 28:219234 .

Skogestad, S. The dos and don'ts of distillation column control. Trans. IChemE, Part A, 2007. 85(A1):1323.

Strand, S. Dynamic Optimization in State-Space Predictive Control Schemes. Ph.D. thesis, Norwegian Institute of Technology (NTH), Trondheim, 1991.

Strand, S. and Sagli, J. MPC in Statoil - Advantages with in-house technology. International Symposium on Advanced Control of Chemical Processes (ADCHEM), Hong Kong, 2004, 2003. pages 97-103.

Tosukhowong, T., Lee, J., Lee, J., and Lu, J. An introduction to a dynamic plant-wide optimization strategy for an integrated plant. Comput. Chem. Eng., 2004. 29:199-208.

Venkat, A., Rawlings, J., and Wright, S. Stability and optimality of distributed, linear model predictive control. Part I: State feedback. Technical report, 2006-03, TWMCC, Department of Chemical Engineering, University of Wisconsin-Madison, 2006.

Ying, C.-M. and Joseph, B. Performance and stability analysis of LP-MPC and QP-MPC cascade control systems. AlChE J., 1999. 45(7):1521-1534. 